\title{
Clinical and Imagistic Aspects in Dog's Hyperadrenocorticism Determined by Pituitary and Adrenal Nodules with Secretory Function
}

\author{
Vladut CIOCIRLAN \\ Tierklinik Keller, 7100 Neusiedl am See, Kellergasse 52, Austria \\ *Corresponding author: office@mypet.ro
}

Bulletin UASVM Veterinary Medicine 72(2) / 2015,

Print ISSN 1843-5270; Electronic ISSN 1843-5378

DOI:10.15835/buasvmcn-vm: 10920

\begin{abstract}
Pituitary dependent hyperadrenocorticism (PDH) is the most common cause of Cushing's syndrome appears in dogs. In approximately $85 \%$ of PDH affected dogs are found by necropsy functional ACTH hormone secreting tumors. Most of the times PDH develops or favors concurrent clinical diseases which occurrence complicating the clinical examination and hamper the diagnosis. Clinical manifestations which motivates the owner to bring the dog to the vet are various and not characteristic for hyperadreno-corticism syndrome. These manifestations take many different forms of disease like diabetes, ascites, nervous symptoms, chronic skin disease and bilateral hair loss, urinary incontinence, liver disease or low back pains. Often excessive secretion of ACTH and cortisol is episodic and causes fluctuations in serum and for a certain period of time can be within normal parameters without triggering clinical signs of disease, and the ACTH stimulation and dexamethasone suppression tests results may be negative.
\end{abstract}

Keywords: diagnostic examinations, dog, pituitary hyperadrenocorticism.

\section{INTRODUCTION}

Hyperadrenocorticism syndrome (Cushing's syndrome) in dogs is a functional disorder which can appear on the differentlevels of the hypotalamopituitary-adrenal axis. Thus pituitary dependent hyperadrenocorticism (PDH) is the most common cause naturally occurring hyperadrenocorticism or Cushing's syndrome in dogs, accounting for $80-85 \%$ of cases (Michael E. Herrtage, 2004). Pituitary dependent hyperadrenocorticism in dogs has various manifestations and not always clearly defined. Diagnosis and differentiation of clinical aspects can be made by complementary clinical examinations (ultrasound, specific hormone level tests, CT). Clinical manifestations are different in dogs with spontaneously appeared hyperadrenocorticism determined by a adrenal tumor (ADH), from those with pituitary hyperadreno-corticism determined by a pituitary tumor, or those with neoplastic processes witch develop adrenal glands metastasis. Most of the nodules which are found after necropsy are small (from $<10 \mathrm{~mm}$ to
$>10 \mathrm{~mm}$ in diameter) and some of them have no secretory function (Peterson et al., 1982). In some cases this nodules have secretory function and they determine an increased ACTH or cortisol secretion over the normal level. Thus, clinical manifestations are dependent on the amount of glucocorticoids secreted in excess and the duration of "intoxication” with those. In dogs with hyperadrenocorticism syndrome caused by pituitary nodules or/and adrenal nodules, the manifestations of this "poisoning” are more „subtle”. In these cases glucocorticoid secretion level is relatively increased but not so much to produce acute changes, detectable in the behavior and the physiology of the dog. In such cases the manifestations are more difficult to be recognized by the owner and often the dogs are presented to veterinarian for various and other apparent simple problems which do not fully complete the clinical picture characteristic for hyperadreno-corticism. In these cases even the specific functional hormonal tests (ACTH stimulation test or dexamethasone 
suppression tests) can be in normal values. In case of adrenal adenomas or adenocarcinomas, cortisol level exceeds by far the normal limits and as result the clinical manifestations will be most of the time acute and easily diagnosed.

\section{MATERIALS AND METHODS}

Theseclinicalmanifestations wereinvestigated in a case of Bichon dog, female, 14 years old, which was presented for five years to the practitioner for breathing problems (coughing and panting), heart and dental problems, light abdominal distension and occasionally digestive problems. The dog had polyuria symptoms, but no polydipsia or polyphagia symptoms. She was under medical supervision and periodically treatment for the last five years (2009-2014), presumptive diagnosed with Cushing's syndrome since 2009. The cortisol level was controlled with ACTH stimulations test when the dog had hyper-adrenocorticism clinical manifestations. The cortisol values after ACTH stimulation test were over the normal limits. The patient became inconstant specific therapy for hyperadrenocorticism clinic manifestations (Vetoryl, Dechra Limited, UK). After five years she was euthanized by owner request because of repeated epileptic seizures. After necropsy it was found pituitary and adrenal nodules.

Examination of the case and radiography imaging investigations were performed in a private clinic with a Compact 30 High Frequency Radiographic device, on digital tapes, read with the help of the Kodak Point-of-Care CR 120 System, in the right lateral decubitus and dorsoventral. The ultrasound investigation was performed with the Mindray Z6 Vet device (Mindray Medical International Limited), with the convex transducer (6C2P), center frequency $6,5 \mathrm{MHz}$, using the real time B mode method, with the animal in the right lateral decubitus and dorsoventral. The blood (CBC and Chemistry Panel) tests were performed in the clinic using Scil Vet abc Plus analyzer (Scil Animal Care Company $\mathrm{GmbH}$ ) and respectively the dry biochemistry machine Fuji Dri-Chem 4000i (Fujifilm Europe $\mathrm{GmbH}$ ). Specific tests like ACTH stimulation test (two blood samples: one for Cortisol base line and the second blood sample at one hour after Synacthen i.v. administration) has been made in the clinic and analyzed by Idexx Laboratory Germany. The urine test (Urine Test Strip) was made in the clinic with Combur 10 Test.

\section{RESULTS AND DISCUSSION}

In the case of the hyperadrenocorticism due to pituitary and adrenal small nodules/adenomas the clinical manifestations are less specific for Cushing's syndrome and are poorly expressed clinical. The pituitary tumors also have the ability to grow and to compress or to invade adjacent structures and to cause nervous symptoms as they develop dorsal towards hypothalamus and thalamus. Some of these nodules/adenomas will determine an excessive secretion of ACTH which will cause bilateral adrenal hyperplasia and excess of cortisol secretion. Excess ACTH secretion will persist despite increased plasma concentrations of cortisol due to lack of the inhibitory mechanism of the ACTH secretion by the cortisol through normal feedback. The most common histological modification is the distal part adenoma, a small percentage is the intermediate part adenoma and in very few cases are detected functional pituitary carcinomas (Michael E. Herritage). A percentage of $50 \%$ of the dogs with PDH have pituitary tumors smaller than 3-5 $\mathrm{mm}$ in diameter and the other half have tumors up to $10 \mathrm{~mm}$ in diameter, particularly those without clinical nervous manifestations, when the pituitary dependent hyperadtrenocorticism (PDH) is diagnosed. A percentage of $10-20 \%$ of dogs has pituitary tumors exceeding $10 \mathrm{~mm}$ in diameter when PDH is diagnosed (Duesberg et al., 1995).

Because of the slightly increased cortisol secretionlevel, higher than thenormallimit,affected dogs will have vague, nonspecific, or intermittent clinical signs (Feldman and Nelson, 2004), mainly chronic problems like dermatological, digestive, dental, eye, ear symptoms or chronic affections of the internal organs (obesity, diabetes).

In our case the dog was presented to the veterinarian for respiratory problems (tracheobronchitis and panting). The general investigation reveals respiratory and heart signs, an intensified vesicular murmur, a chronic oral and ocular inflammation (gingivitis and conjunctivitis and mature cataract), secondary an increased sensitivity in tonsil region. The general appearance was like overweight, tired dog with moderate decrease in muscle tonus, abdomen moderat increased in volume, sensitive to touch, appetite present but not overly exceeded, relative increased water consumption but not permanent. On the skin, at the abdominal and perineal level was 
found erythematous dermatitis and pigmented skin areas. The dog had the same diet over the past few months.

The blood count results shown moderate leukocytosis, monocytosis, granulocytosis (WBC $17.710^{3} / \mu \mathrm{L}$, MON $0.610^{3} / \mu \mathrm{L}$, GRA $15.010^{3} /$ $\mu \mathrm{L})$, regenerative anemia (RBC $6.1710^{3} / \mu \mathrm{L}, \mathrm{HGB}$ $13.4 \mathrm{~g} / \mathrm{dL}$, HCT $42.3 \%$, MCV $68 \mu \mathrm{m}^{3}$, MCH 21.8 pg, MCHC $31.8 \mathrm{~g} / \mathrm{dL}$, PLT $1110^{3} / \mu \mathrm{L}$, MPV 11.5 $\mu \mathrm{m}^{3}$ ) and the blood chemistry a moderate uremia (BUN $31.0 \mathrm{mg} / \mathrm{dl}$, IP $5.2 \mathrm{mg} / \mathrm{dl}$, CRE $0.9 \mathrm{mg} / \mathrm{dl}$, GPT 26 U/l, GOT 22 U/l, ALP 50 U/l, Ca mg/dl). The radiological examination in the right lateral recumbency reveal an overly enlarged heart, generally slightly distended abdomen, lack of abdominal muscle tone, hepatomegaly, that far exceeds the costal arch, splenomegaly, slightly caudal kidneys, small urinary bladder calculus and fat deposits in the lumbar region (Fig. 1).

The ultrasound examination preformed in the right lateral and dorsal recumbency could reveal

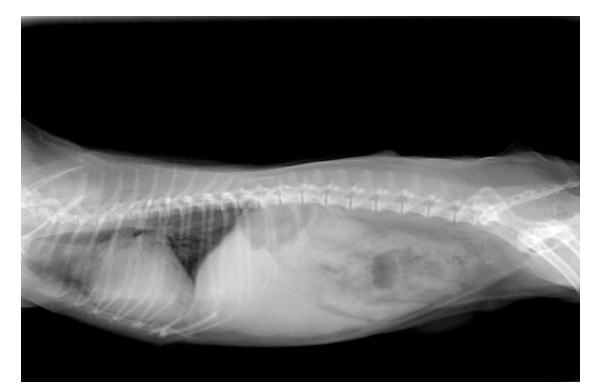

Fig. 1. Radiological assess in right lateral view, Bichon, 14 years, female. We can assess the overly enlarges heart, the abdominal distension and the size of the liver which overcome the last rip, splenomegaly and a small urinary bladder calculus.

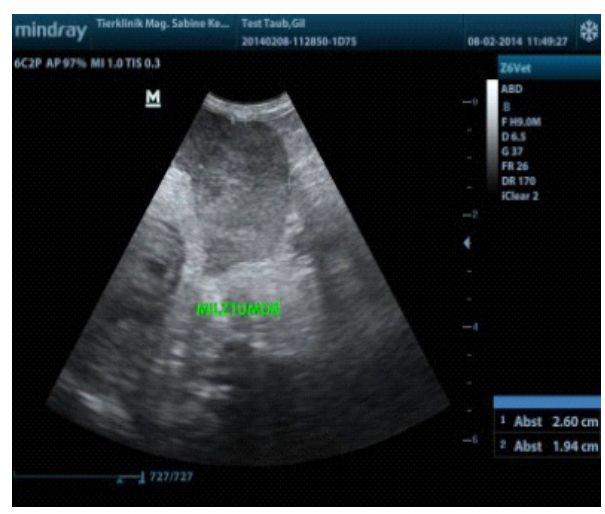

Fig. 3. Ultrasonographic assess of the spleen. It can be seen the presence of splenic mases. the presence of multiple nodular formations in the abdominal organs structure.

In the Fig. 2, 3 and 4 can be observed metastases that have developed in the last few years in liver, in spleen that caused deformation of the spleen capsule, with a hypoechoic-anechoic aspect, and at the extracapsular renal level. The ultrasound examination of the adrenal glands revealed the nodular appearance of the left adrenal gland with irregular outline and hypoechoic nonhomogeneous aspect; it preserved the „peanut” shape and doesn't exceed too much the normal limits (Fig. 5). On the right side, the adrenal gland has a hypoechoic aspect with a posterior acoustic shadow, keeps the characteristic "comma" shape and is slightly enlarged in volume (diameter $2.19 \mathrm{~cm}$ long, normally up to $2 \mathrm{~cm}$ ) with nodular appearance and irregular margins. Both adrenal glands are relatively increased in volume, not much above the normal range, with irregular shape due to the presence of nodules at this level.

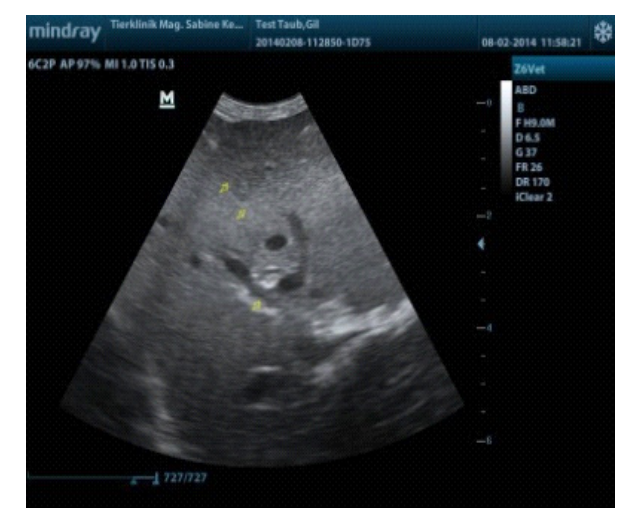

Fig. 2. Ultrasonographic liver assess. It can be seen an hypoechoic area on the left lobe.

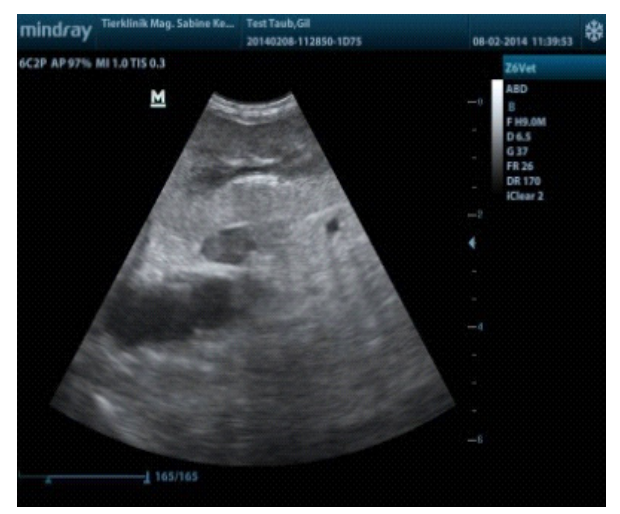

Fig. 4. Ultrasonographic assess of the left kidney with extracapsular metastase. 


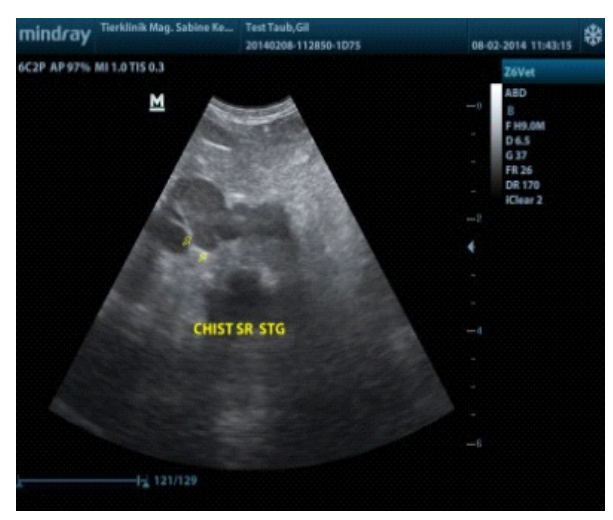

Fig. 5. Ultrasonographic aspect of left adrenal gland nodul.

The result of ACTH stimulation test was positive: cortisol baseline level is $8.0 \mu \mathrm{g} / \mathrm{dL}$ (normal 0.5-6.5 $\mu \mathrm{g} / \mathrm{dL}$ ) and after stimulation 23.3 $\mu \mathrm{g} / \mathrm{dL}$ (normal $<15.0 \mu \mathrm{g} / \mathrm{dL}$ ). The cortisol level has stabilized after specific treatment.

In this case symptoms were intermittent and vague, the general manifestations showed an incomplete clinical panel with hyperadrenocorticoticism syndrome. The vague clinical signs and particular imagistic aspects (Röntgen and Ultrasound) were completed with blood tests and special adrenal function test (ACTH stimulation test) for diagnosis.

In these cases slightly higher than normal cortisol secretion did not caused complete characteristic Cushing's syndrome symptoms panel. Because of the secretors pituitary nodules, ACTH secretion is increased and no longer reacts to the increased amount of cortisol in the blood by negative feedback, which gradually lowers immunity and causes chronic skin, liver, digestive, urinary and metabolic problems. Presumptive diagnosis was established due to clinical aspects and imagistic examination (Roentgen and Ultrasound exams) and confirmed due blood test and blood cortisol level tests. The patient had intermittent relapses over five years. It was observed that in time the symptoms were more and more characteristic with hyper-adrenocorticism. In the last year the dog had also developed nervous symptoms, epileptic like crises and the dog was euthanized by owner request.

Following necropsy examination could be revealed the presence of micronodules at the pituitary and adrenal glands level.

\section{CONCLUSION}

Hyperadrenocorticism in dogs determined by pituitary and adrenal nodules with secretory function can evolve over many months or even years. Clinical manifestations are vague and hamper diagnosis. The cortisol level can be slightly over the normal limits. Radiological we can have the general aspect changes similar to other hepatitis, diabetes and ascites and by ultrasound it can be found the adrenal glands modifications (relatively increased in volume or nodules).

A presumptive diagnosis can be made with the imagistic exam (Roentgen and Ultrasound) and blood tests (CBC, parameters checkup, ACTH stimulation test). In addition it can be found, most often changes due to associated diseases. Because inhibition of the immune system, often this type of Cushing develops in association with other diseases (dermatitis, kidney diseases, pancreatic diseases, tumors or diffuse cancers). The cortisol level determination after ACTH stimulation tests or dexamethasone suppression tests can be normal at the beginning of disease. The characteristics aspects of Cushing's syndrome occur later, after a relatively long period of time and the concurrent clinical changes looks like chronic symptoms and slowly healing after treatment but periodically relapsed.

\section{REFERENCES}

1. Barthez PY, Nyland TG, Feldman EC (1995). Ultrasonographic evaluation of adrenal glands in dogs. J Am Vet Med Assoc 207:1180-1183.

2. Behrend ED, Kemppainen RJ, Clark TP, Salman MD, Peterson ME (2002). Diagnosis of hyperadrenocotricism in dogs: A survey of internists and dermatologists. J Am Vet Med Assoc 220:1643-1649.

3. Douglass JP, Berry CR, James S (1997). Ultrasonographic adrenal gland measurements in dogs without evidence of adrenal disease. Vet Radiol Ultrasound 38:124-130.

4. Feldman EC (1983). The adrenal cortex. In : Ettinger SJ (ed). Textbook of Veterinary Internal Medicine, 2nd ed, WB Saunders Co, Philadelphia.

5. Feldman EC, Nelson RW (2004). The adrenal gland. In : Feldman EC, Nelson RW (eds). Canine and Feline Endocrinology and Reproduction, 3rd ed, WB Saunders, St Louis, 394-439.

6. Grooters Am, Biller DS, Merryman J (1995). Ultrasonographic parameters of normal canine adrenal glands: Comparison to necropsy findings. Vet Radiol Ultrasound 36:126-130.

7. HoeraufA, Reusch C (1999). Ultrasonographic evaluation of the adrenal glands in six dogs with hyperadrenocorticism. J Am Anim Hosp Assoc 35:214-218. 
8. Kealy JK, McAlister H (2000). Diagnostic Radiology and Ultrasonography of the Dog and Cat, 3rd ed. WB Saunders Co, Philadelphia.

9. Mooney TC, Mark EP (2004). BSAVA Manual of Canine and Feline Endocrinology, 3rd ed, British Small Animal Veterinary Association, Gloucester.
10. Penninck D, d`Anjou MA (2008). Atlas of Small Animal Ultrasonography. Blackwell Publishing, 385-395. 